\title{
Algunas precisiones acerca de la mortalidad por enfermedades cardiovasculares en Cuba
}

\author{
Some clarifications about the mortality from cardiovascular \\ diseases in Cuba
}

\author{
Armando H. Seuc, ${ }^{\text {I }}$ Emma Domínguez Alonso, ${ }^{\text {II }}$ Rosa María Torres Vidal, ${ }^{\text {III }}$ \\ Patricia Varona Pérez ${ }^{\mathrm{IV}}$ \\ ILicenciado en Matemática. Doctor en Ciencias Matemáticas. Instituto de Higiene, \\ Epidemiología y Microbiología (INHEM). La Habana, Cuba. \\ IIDoctor en Medicina. Especialista de II Grado en Bioestadística. Instituto Nacional \\ de Endocrinología (INEN). La Habana, Cuba.

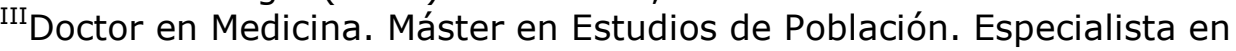 \\ Bioestadística. Dirección Nacional de Registros Médicos y Estadísticas de Salud \\ (DNE), MINSAP. La Habana, Cuba. \\ IV Doctor en Medicina. Investigador Auxiliar. Instituto Nacional de Higiene, \\ Epidemiología y Microbiología (INHEM). La Habana, Cuba.
}

\section{RESUMEN}

Introducción Las enfermedades del corazón siguen siendo la primera causa de muerte en Cuba. El impacto de esta mortalidad en términos de Años de Vida Potencial Perdidos por Muertes Prematuras ha sido poco abordado a nivel nacional. Objetivos Describir la mortalidad y su impacto sobre la esperanza de vida (expresado en Años de Vida Potencial Perdidos por Muertes Prematuras) en Cuba en los años 1990, 1995, 2000 y 2005, para un grupo de sub-causas dentro de las enfermedades del corazón.

Métodos Se trabajó con los registros de mortalidad de la Dirección Nacional de Estadísticas de los años citados, las tasas se ajustaron por sexo y edad por el método directo con respecto a 1990. Los Años de Vida Potencial Perdidos por Muertes Prematuras, se calcularon para grupos de edades quinquenales y usando la esperanza de vida para el año 2000. Las enfermedades del corazón se desglosaron en cinco sub-causas.

Resultados El infarto agudo del miocardio fue la primera causa de muerte en el periodo estudiado, con tendencia importante al decremento, mientras que, "otras enfermedades isquémicas", fue la segunda causa con tendencia ligera al 
incremento. La brecha en las tasas de mortalidad según sexo en Cuba es menor que la informada en diversos países desarrollados, en particular para enfermedades isquémicas del corazón, existen potencialidades no totalmente aprovechadas para reducir aún más la mortalidad en mujeres. De 1990 al 2000 se produjeron disminuciones apreciables en las tasas de mortalidad por distintas sub-causas dentro de las enfermedades del corazón, sin embargo a partir del 2000 las correspondientes tasas se han estabilizado o han recuperado una tendencia al incremento.

Conclusiones Se sugiere seguir el comportamiento de la mortalidad no solo por infarto agudo de miocardio sino también por otras enfermedades isquémicas del corazón y explorar las posibilidades de reducir aún más la mortalidad en mujeres.

Palabras clave: Enfermedades del corazón, carga de enfermedades, mortalidad, Cuba.

\section{ABSTRACT}

Introduction Heart diseases are still the first cause of death in Cuba. The impact of cardiovascular mortality in terms of Potential Lost Life Years from Premature Death has not been extensively addressed nationwide.

Objectives to describe the mortality and the impact on the life expectancy (expressed as Potential Lost Life Years from Premature Death) in Cuba in 1990, 1995, 2000 and 2005, for a group of heart disease-related sub-causes.

Methods The study used the mortality records of the National Division of Statistics in the above-mentioned years, the rates were sex- and age-adjusted by the direct method in comparison to 1990 . The potential lost life years from premature death were estimated for five-year period age groups by using life expectancy for the year 2000. The heart diseases were broken down to five sub-causes.

Results The acute myocardial infarct was the first cause of death in the studied period, with important tendency to decrease whereas "other ischemic diseases" was the second cause tending to slight increase. The gap in the mortality rates by sex in Cuba is lower than that reported in several developed countries, particularly in ischemic heart diseases; there are some underutilized potentialities for further reduction of female mortality. In the 1990-2000 period, sizeable reductions in mortality rates per various heart disease sub-causes were reached; however, the respective mortality rates have stabilized or have returned to an increasing tendency from 2000 on.

Conclusions It is suggested that mortality from other heart ischemic diseases be considered together with the mortality from acute myocardial infarct and that possibilities to reduce even more female mortality be explored.

Key words: Heart diseases, disease burden, mortality, Cuba.

\section{INTRODUCCIÓN}

Numerosos trabajos en Cuba han abordado el importante tema de las enfermedades cardiovasculares (ECAV) en los últimos años. ${ }^{1-15}$ Solo uno de ellos

http://scielo.sld.cu 
aborda en detalle, con alcance nacional, la mortalidad por ECAV y sus causas componentes, ${ }^{1}$ el resto se concentra en la incidencia/prevalencia de causas y factores de riesgo relacionados, ${ }^{3,4,6-10,13}$ o en una causa específica dentro de las ECAV o en un grupo poblacional específico, ${ }^{2,5,12}$ otros abordan solo la mortalidad por ECAV sin desglose, ${ }^{11}$ (Seuc AH. Evolution of Cuban Disease Burden at 5-year intervals: 1990-2005. Pendiente de publicación) o son artículos de revisión. ${ }^{14,15}$

De lo anterior pareciera que la mortalidad y su impacto son asuntos agotados en el país. En realidad, consideramos que dada la notoriedad de las ECAV el tema debiera ser motivo de más y mejores estudios por la comunidad científica cubana.

El propósito de este trabajo es tratar de presentar un cuadro, lo más preciso posible, de la mortalidad en Cuba por ECAV en los últimos años y del impacto de esta mortalidad sobre la esperanza de vida, para lo cual nos apoyaremos fundamentalmente en el artículo de Ordúñez $P .^{1}$ Esperamos que esta descripción detallada sea de alguna utilidad para la elaboración de estrategias que persigan el mejoramiento de la salud de la población cubana, tal y como lo ha sido a nivel global. ${ }^{16}$

\section{MÉTODOS}

Para este trabajo se utilizaron las bases de datos de mortalidad de la Dirección Nacional de Estadísticas (DNE) del MINSAP, de los años 1990, 1995, 2000 y 2005. Se calcularon las tasas de mortalidad $(\times 100000)$ y de Años de Vida Potencial Perdidos por Muertes Prematuras (AVPP $\times 10$ 000) para las siguientes causas dentro de la categoría "Enfermedades cardiovasculares" (ECAV):

1. Infarto agudo del miocardio (IAM). CIE 9na.: 410; CIE 10ma.: I21-I22.

2. Otras enfermedades isquémicas del corazón (OEI). CIE 9na.: 411-414; CIE 10ma.: I20, I23-I25.

3. Enfermedades reumáticas del corazón (ER). CIE 9na.: 393-398; CIE 10ma.: I05I09.

4. Enfermedades inflamatorias del corazón (EI). CIE 9na.: 420-422; CIE 10ma.: I30-I33, I38, I40, I42.

5. Enfermedades hipertensivas del corazón (EH). CIE 9na.: 401-405; CIE 10ma.: I10-I15.

6. Enfermedades cerebrovasculares (ECEV). CIE 9na.: 430-438; CIE 10ma.: I60I69.

7. Enfermedades de las arterias, arteriolas y vasos capilares (AAVC). CIE 9na.: 440-448; CIE 10ma.: I70-I79.

Las causas de la 1 a la 5, junto con "otras enfermedades del corazón" (CIE-9na.: 415-419, 423-429; CIE-10ma.: I26-I29, I34-I37, I39, I41, I43-I52) conformaron lo que denominamos "Enfermedades del corazón" (ECO), categoría de uso frecuente en los Anuarios Estadísticos de Salud cubanos. Las categorías 1 y 2 conformaron lo

http://scielo.sld.cu 
que se denomina "Enfermedades isquémicas (o coronarias) del corazón" (EIC), de frecuente uso en estudios internacionales. La gran categoría "Enfermedades cardiovasculares" se organizaron con los códigos CIE-9na.: 390-459, y CIE-10ma.: I00-I99.

Las tasas de mortalidad se ajustaron por edad y sexo con respecto a la población de Cuba en 1990 usando el método directo; no se ajustó con respecto a la población de 1981 (referencia usual en los Anuarios Estadísticos de Salud) pues no estaba disponible. Los AVPP se calcularon sin descuento en el tiempo y sin ponderación por edad, usando como referencia la esperanza de vida en Cuba para el año $2000 .^{17}$

Se calcularon también tasas específicas por grupos de edad; los grupos de edad empleados fueron: 0 a 29, 30 a 49, 50 a 59, 60 a 69, 70 a 79, y 80 o más.

\section{RESULTADOS}

Las tablas 1,2 y 3 muestran los resultados de las tasas de mortalidad ( $\times 100000$ ) y de las tasas de AVPP ( $\times 10000$ ), para población total, hombres, y mujeres. Los resultados se muestran para las siete causas de muerte relacionadas en la sección previa, y para algunos subtotales. Las figuras 1 y 2 muestran las tasas de mortalidad y de AVPP para ambos sexos

Tabla 2. Hombres. Enfermedades cardiovasculares: 1990, 1995, 2000 y 2005. Tasas ajustadas por edad, método directo, población de referencia año 1990. Tasas mortalidad ( $\times 100$ 000) y tasas AVPP (X 10000 )

\begin{tabular}{|l|r|r|r|r|r|r|r|r|r|}
\hline \multirow{2}{*}{ Enfermedades } & \multicolumn{2}{|c|}{1990} & \multicolumn{2}{|c|}{1995} & \multicolumn{2}{|c|}{2000} & \multicolumn{2}{|c|}{2005} \\
\cline { 2 - 9 } & Mort. & AVPP & Mort. & AVPP & Mort. & AVPP & Mort. & AVPP \\
\hline Inf. agudo miocardio (IAM) & 141,1 & 193,1 & 114,1 & 158,4 & 72,0 & 112,1 & 58,4 & 89,1 \\
\hline Otras enf. isq. corazón(OEI) & 49,4 & 46,4 & 46,2 & 43,4 & 49,4 & 50,7 & 56,0 & 56,6 \\
\hline Enf. reumáticas corazón (ER) & 1,4 & 4,6 & 1,3 & 4,1 & 1,6 & 4,3 & 0,9 & 2,5 \\
\hline Enf. inflamatorias corazón (EI) & 0,4 & 1,4 & 1,0 & 5,2 & 0,5 & 2,1 & 5,0 & 12,8 \\
\hline Enf. hipertensivas (EH) & 7,6 & 12,6 & 8,7 & 14,4 & 9,8 & 16,3 & 12,0 & 17,4 \\
\hline Enf. cerebrovasculares (ECEV) & 65,5 & 89,3 & 61,0 & 78,1 & 56,6 & 72,7 & 56,8 & 71,4 \\
\hline Arterias, arteriolas, VC (AAVC) & 27,8 & 25,2 & 29,8 & 26,8 & 26,7 & 24,5 & 21,2 & 20,7 \\
\hline Enf. cardiovasculares (ECAV) & 314,6 & 403,1 & 288,4 & 366,5 & 244,1 & 321,8 & 236,7 & 302,3 \\
\hline Enf. del corazón (ECO) & 199,9 & 258,1 & 171,3 & 225,5 & 133,3 & 185,4 & 132,2 & 178,4 \\
\hline Enf. isquémicas corazón (EIC) & 190,5 & 239,5 & 160,4 & 201,8 & 121,4 & 162,8 & 114,4 & 145,7 \\
\hline
\end{tabular}

http://scielo.sld.cu 
Tabla 3. Mujeres. Enfermedades cardiovasculares: 1990, 1995, 2000 y 2005 Tasas ajustadas por edad, método directo, población de referencia año 1990. Tasas mortalidad ( $\times 100$ 000) y tasas AVPP ( 10000 )

\begin{tabular}{|c|c|c|c|c|c|c|c|c|}
\hline \multirow{2}{*}{ Enfermedades } & \multicolumn{2}{|c|}{1990} & \multicolumn{2}{|c|}{1995} & \multicolumn{2}{|c|}{2000} & \multicolumn{2}{|c|}{2005} \\
\hline & Mort. & AVPP & Mort. & AVPP & Mort. & AVPP & Mort. & AVPP \\
\hline Inf. agudo miocardio (IAM) & 104,4 & 137,4 & 79,0 & 102,8 & 44,1 & 60,7 & 37,2 & 53,1 \\
\hline Otras enf, isq, corazón(OEI) & 47,7 & 46,6 & 44,7 & 44,4 & 44,7 & 46,4 & 50,6 & 51,5 \\
\hline Enf, reumáticas corazón (ER) & 2,3 & 8,8 & 1,6 & 5,5 & 1,8 & 6,0 & 1,4 & 4,1 \\
\hline Enf, inflamatorias corazón (EI) & 0,5 & 1,9 & 0,7 & 4,2 & 0,4 & 1,6 & 2,0 & 6,6 \\
\hline Enf, hipertensivas (EH) & 8,0 & 13,9 & 7,9 & 13,2 & 8,9 & 14,2 & 10,1 & 15,4 \\
\hline Enf. cerebrovasculares (ECEV) & 65,3 & 93,9 & 63,0 & 86,2 & 56,0 & 75,9 & 54,4 & 69,3 \\
\hline Arterias, arteriolas, VC (AAVC) & 26,0 & 22,6 & 24,8 & 22,1 & 21,6 & 20,3 & 17,3 & 16,6 \\
\hline Enf. cardiovasculares (ECAV) & 274,6 & 356,0 & 245,1 & 312,5 & 200,5 & 257,3 & 197,4 & 247,1 \\
\hline Enf. del corazón (ECO) & 162,9 & 208,6 & 134,0 & 170,1 & 99,9 & 129,0 & 101,3 & 130,7 \\
\hline Enf. isquémicas corazón (EIC) & 152,1 & 184,0 & 123,7 & 147,2 & 88,8 & 107,1 & 87,8 & 104,6 \\
\hline
\end{tabular}

La tabla 4 muestra las tasas de mortalidad para los seis grupos de edad descritos en Métodos, para las causas IAM, EIC, ECO y ECAV. Las figuras 3 y 4 muestran los resultados de la tabla 4 para el caso de ECO.

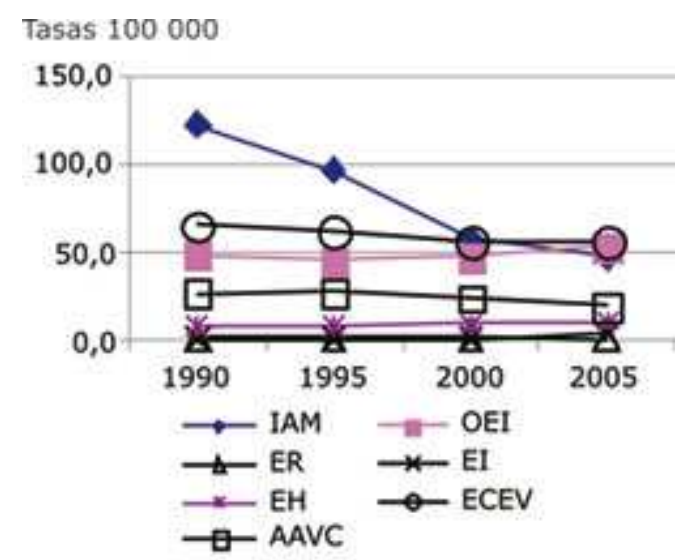

Fig. 1. Tasas mortalidad. Total.

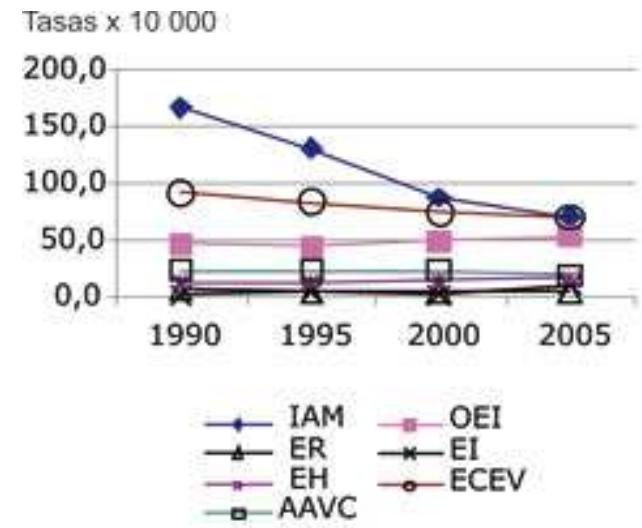

Fig. 2. Tasas AVPP. Total. 
Tabla 4. Tasas de mortalidad ( $\times 100000$ ) por enfermedades cardiovasculares para hombres y mujeres con edades entre 35 y 74 años

\begin{tabular}{|l|c|c|c|r|r|r|r|}
\hline \multirow{2}{*}{ País } & \multicolumn{3}{|c|}{ Hombres } & \multicolumn{3}{c|}{ Mujeres } \\
\cline { 2 - 8 } & ECAV & EIC & ECEV & ECAV & EIC & ECEV \\
\hline EE. UU. (2003) & 367 & 187 & 36 & 158 & 77 & 28 \\
\hline Inglaterra/Gales (2002) & 301 & 196 & 49 & 138 & 68 & 36 \\
\hline Australia (2001) & 206 & 138 & 31 & 92 & 47 & 22 \\
\hline Canada (2001) & 222 & 150 & 29 & 97 & 51 & 21 \\
\hline Cuba (2000) + & 244 & 121 & 57 & 200 & 89 & 56 \\
\hline
\end{tabular}

Fuente: World health statistics 2006. Geneva: World Health Organization; 2006. Disponible en: www. who. int/whosis/whostat2006.pdf (citado 19 Dic 2006). ECAV: Enfermedad cardiovascular; EIC: Enfermedad isquémica del corazón; ECEV: Enfermedad cerebrovascular; t: tasa para todas las edades

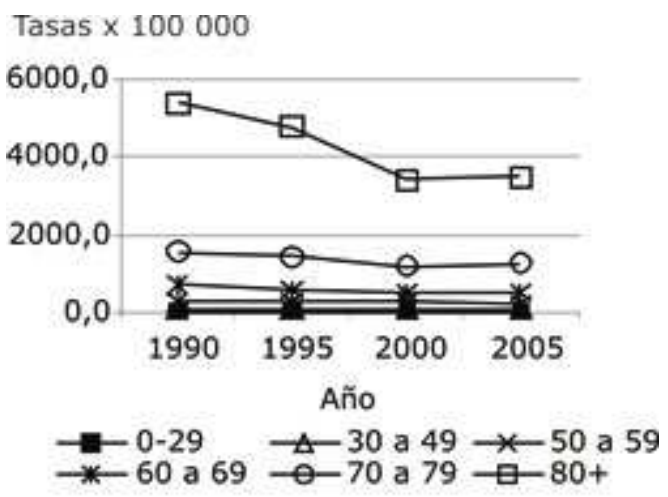

Fig. 3. Tasa mortalidad corazón, por grupos de edad. Hombres.

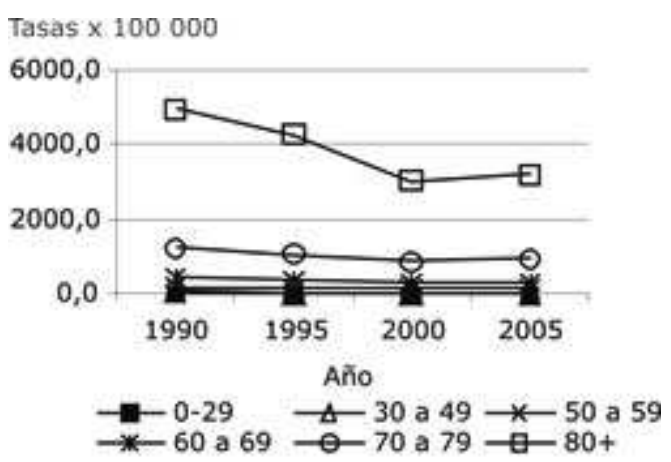

Fig. 4. Tasas mortalidad corazón, por grupos de edad. Mujeres.

\section{Mortalidad}

En los resultados destaca el comportamiento de IAM que, tanto en mujeres como (sobre todo) en hombres, tuvo disminuciones importantes en sus tasas de mortalidad durante el periodo estudiado. La brecha entre hombres y mujeres se ha ido cerrando en términos absolutos; en 1990 la diferencia entre las dos tasas era de 36,7 ( $\times 100000)$, mientras que en el 2005 era de $21,2(\times 100000)$. En

http://scielo.sld.cu 
términos relativos, sin embargo, la brecha ha aumentado; en 1990 había cerca del $35 \%$ más de mortalidad en hombres que en mujeres, mientras que en el 2005 la correspondiente cifra fue de $57 \%$.

Este comportamiento favorable de IAM contrastó con el de OEI, que mostró ligeros pero sostenidos incrementos de sus tasas de mortalidad a partir de 1995, similares en hombres y en mujeres. Semejante al comportamiento de OEI fue el de EH, que ya desde 1990 mostró ligeros pero sostenidos incrementos de sus tasas de mortalidad.

Las ECEV tuvieron un comportamiento ligeramente favorable (tasas alrededor de 60 $\times 100000$ ) durante el periodo estudiado, observándose en general reducciones modestas tanto en hombres como en mujeres. AAVC mostró reducciones ligeras en sus tasas de mortalidad, sobre todo en mujeres y a partir de 1995.

Con el paso del tiempo se observó que ha ido disminuyendo la variabilidad de la mortalidad entre las siete causas consideradas. Como consecuencia de este comportamiento, en el 2005 las tasas de mortalidad por IAM, ECEV y OEI en hombres fueron prácticamente las mismas (alrededor de 57), mientras que en 1990 la diferencia entre IAM y OEI fue de 92; en mujeres, en el 2005 pasaron a ocupar los dos primeros lugares en mortalidad ECEV y OEI, quedando algo cerca y por debajo IAM. Todo parece indicar que, si se mantuvieron las tendencias observadas en el periodo 1990-2005, después del 2005 y hasta la fecha, la principal causa de muerte en Cuba dentro de las ECAV pasó a ser OEI, seguida de cerca por ECEV en 2do. lugar e IAM en 3er. lugar.

Las tasas de mortalidad por EI se mantuvieron muy bajas en el periodo, aunque en el 2005, tanto en hombres como en mujeres, tuvieron incrementos importantes. Las tasas de mortalidad por ER continuaron muy bajas en el periodo, destacándose el hecho de que siempre han sido mayores en mujeres que en hombres.

Globalmente, las ECO mostraron una disminución en sus tasas desde 1990 hasta el 2000, con un ligero repunte entre el 2000 y el 2005. Las ECAV en bloque tuvieron una disminución sostenida hasta el 2000, con una casi estabilización entre el 2000 y el 2005.

\section{Años de Vida Potencialmente Perdidos}

El comportamiento comparativo de las distintas ECAV a lo largo del periodo 19902005, desglosado por sexo, mostró una casi total correspondencia entre los patrones observados en las tasas de AVPP y las tasas de mortalidad.

El IAM en hombres generó en el periodo la mayor cantidad de AVPP, aunque en el 2005 su impacto negativo sobre la esperanza de vida se acercó mucho al generado por ECEV y OEI. En mujeres la afectación principal sobre la esperanza de vida la ejerció el IAM hasta poco antes del 2000 aproximadamente, a partir de ese momento la afectación principal pasó a ser generada por las ECEV.

Las tendencias observadas en las tasas de mortalidad descritas en la sección previa se aplicaron, (casi) sin excepción, a las tasas de AVPP. En particular, se reiteró el hecho de que a partir de 1995 se registraron incrementos ligeros pero sostenidos en los AVPP debido a OEI; de mantenerse esta tendencia es posible que ya en el año 2009, o dentro de pocos años, los AVPP originados por OEI sean la principal causa de afectación a la esperanza de vida dentro de todas las ECAV en Cuba, tanto en hombres como en mujeres.

http://scielo.sld.cu 


\section{DISCUSIÓN}

Los valores absolutos de las tasas de mortalidad presentadas en este artículo difieren en alguna medida de las registradas en los Anuarios Estadísticos de Salud cubanos, ${ }^{18,19}$ y por Orduñez, ${ }^{1}$ debido a que en el primer caso estandarizamos con respecto a la población cubana de 1990, mientras que en los restantes casos las tasas usadas fueron estandarizadas con respecto a la población cubana de 1981. Esto sin embargo, no afecta ninguna de las comparaciones realizadas, ni entre las distintas causas de muerte ni a lo largo del periodo estudiado 1990-2005.

Ordúñez ${ }^{1}$ reproduce las 10 primeras causas de muerte para Cuba en el 2003 a partir del Anuario Estadístico de Salud de la DNE-MINSAP para ese año. ${ }^{20}$ Las causas de muerte que aparecen en una relación de este tipo dependen por supuesto de cuál es el listado inicial de causas que se están considerando. Lo que valoramos como la omisión más importante en ese listado, obviando diversidades en la (re)agrupación de las Enfermedades Obstructivas Crónicas (incluyendo o no "asma" y "bronquitis y enfisema") y de las "Enfermedades Digestivas" (incluyendo o no "cirrosis y otras crónicas del hígado"), es "Condiciones Neuropsiquiátricas", que en el 2000 era la $7 \mathrm{ma}$. causa de muerte más importante en ambos sexos, y entre la 6ta. y la 7ma. causa más importante de AVPP, en mujeres y hombres respectivamente, ${ }^{11}$ situación similar se presentó en el 2005 (Seuc AH. Evolution of Cuban Disease Burden at 5-year intervals: 1990-2005. Pendiente de publicación).

Como se señala, ${ }^{1}$ ECO era en el 2003, y sigue siendo, ${ }^{21}$ la primera causa de muerte en Cuba, pero debiera precisarse que seguida cada vez más de cerca por Cáncer, la cual sobrepasó a ECO en AVPP ya desde 1999 (Domínguez E. Carga de las Enfermedades en Cuba. Comunicación personal, 2002). Es decir, que la causa que más está afectando la esperanza de vida en el país desde 1999 dejó de ser ECO y pasó a ser Cáncer.

Esta preponderancia del Cáncer es, sin embargo, controversial. A pesar de que internacionalmente es una causa de muerte de uso común en los estudios de mortalidad y su impacto, Cáncer es una causa de muerte muy heterogénea en lo que a sus factores de riesgo se refiere, mucho más que ECO o incluso ECAV, por lo que desde los puntos de vista de prevención y promoción de salud no resulta de mucha utilidad hablar del Cáncer como una causa de muerte importante.

Orduñez $P_{1}^{1}$ plantea que la segunda categoría diagnóstica más frecuente en el 2003 dentro del grupo de ECO es EH. Sin embargo, si consideramos las EIC excluyendo IAM, es decir OEI, esta última es ampliamente la segunda categoría diagnóstica más frecuente, tanto en hombres como en mujeres, tanto en mortalidad como en AVPP, y durante todo el periodo $1990-2005$ (tablas 1,2 y 3 ).

Siguiendo con la mortalidad por EIC hemos notado que algunos trabajos nacionales publicados en el $2001,{ }^{14,15}$ hacen referencia a que 1 de cada 4 muertes ( $25 \%$ ) en el país se producían (pensamos que en el 2001 o en el 2000 pues no se especifica) por esta causa. En ese sentido nuestros resultados y los de los correspondientes Anuarios Estadísticos de Salud muestran que las proporciones son 25, 23, 20 y 19 $\%$ para 1990, 1995, 2000 y 2005, respectivamente, luego estrictamente hablando, decir que 1 de cada 4 muertes en Cuba se producían por EIC es válido solo para el año 1990 (o antes de 1990 quizás).

Se plantea que las ER han prácticamente desaparecido, ${ }^{1}$ con lo cual coincidimos, pero no se hace mención de las EI. Entre 1990 y 2000 las EI tuvieron las más bajas

http://scielo.sld.cu 
tasas de mortalidad y de AVPP dentro de todas las cinco ECO consideradas en este trabajo; sin embargo, del 2000 al 2005 incrementaron sus tasas de mortalidad y de AVPP en 5 y en 4 veces respectivamente, con respecto a sus valores promedios durante 1990-2000 (tabla 1).

Orduñez, ${ }^{1}$ declara,a partir del Anuario Estadístico de Salud del $2003,{ }^{20}$ que la tasa de AVPP por ECAV en Cuba en el 2003 era de 11 ( $\times 1$ 000). Debemos decir que esta cifra es incorrecta por dos razones, en primer lugar, pensamos que este autor, ${ }^{1}$ se está refiriendo a la tasa de AVPP por ECO y no por ECAV, ya que los Anuarios Estadísticos de Salud cubanos no reportan AVPP para ECAV. En segundo lugar, el Anuario correspondiente al $2003,{ }^{20}$ en su tabla 15, presenta también los AVPP usando una referencia fija (esperanza de vida al nacer), independientemente de la edad a la cual ocurría la muerte, lo que es metodológicamente inadecuado pues, entre otros problemas, de esta forma se subestima mucho y sin necesidad la verdadera afectación a la esperanza de vida que los AVPP representan. Una mejor estimación para esta tasa de AVPP

( $\times 1000$ para hacerla comparable) debido a ECAV para el 2000 es 22,2, mientras que para el 2005 es 21,7 (tabla 1), es decir, 2 veces mayor que la registrada por Ordúñez, ${ }^{1}$ esto puede verificarse finalmente en el Anuario Estadístico de Salud del $2003,{ }^{20}$ donde la tasa ( $\times 1$ 000) de AVPP para ECO usando la esperanza de vida a la edad en que ocurrían las muertes es de 24,6 .

El autor, ${ }^{1}$ señala también que las tasas de mortalidad por EIC en hombres y en mujeres han tenido el mismo patrón a lo largo del tiempo (1970-2003), aunque las tasas en hombres han estado siempre por encima de las tasas en mujeres. Si bien, lo que se ha observado en los resultados de este trabajo para el periodo 1990-2005 coincide en general con esa afirmación, el análisis de IAM, la causa más importante dentro de EIC, muestra una reducción en sus tasas más pronunciada en hombres que en mujeres, además de que los hombre parten (en 1990) de tasas significativamente más altas que las de las mujeres (tablas 1, 2 y 3); esta asimetría ha sido motivo de preocupación en algunos trabajos recientes publicados en la literatura internacional. ${ }^{22,23}$

La comparación de nuestras tasas de mortalidad por ECAV y por EIC con las de varios países desarrollados, presentada en una publicación de la Organización Mundial de la Salud en el $2006,{ }^{24}$ muestra que los valores tienen cierta similitud en hombres, sin embargo en mujeres nuestras tasas son significativamente más altas que las de esos países (tabla 4); esto reitera la existencia en Cuba de una brecha notablemente menor entre hombres y mujeres que la brecha usualmente encontrada en países desarrollados en lo relativo a ciertos indicadores como mortalidad y esperanza de vida, lo que podría ser reflejo de que hemos tenido potencialidades en la promoción, prevención, tratamiento en mujeres que no hemos aprovechado suficientemente.

En la sección "Resultados" se ha comentado que es inusual que la importante reducción en las tasas de IAM durante todo el periodo 1990-2005 se acompañara de ligeros incrementos en las tasas de OEI a partir de 1995 (tabla 1, figura 2). Esta "anomalía" hace pensar inevitablemente en la posibilidad de que algunas muertes por IAM se estén "desplazando" incorrectamente hacia algunas causas dentro de OEI, lo que habría que corroborar con estudios específicos de validación.

Finalmente, Ordúñez $y_{1}{ }^{1}$ plantea que las tasas de mortalidad específicas por grupos (quinquenales) de edad para ECAV muestran en general un comportamiento que se caracteriza por incrementos desde mediados de los 70 hasta finales de los $80, y$ "una franca disminución desde inicios de los 90"; en ${ }^{1}$ se trabaja con grupos quinquenales entre 50 y 79 años.

http://scielo.sld.cu 
Nuestro análisis, que comienza en 1990, encuentra las reducciones más apreciables en las tasas de mortalidad para el grupo de edad "80+",no considerado por Orduñez, ${ }^{1}$ seguidas por las reducciones en el grupo de edad "70 a 79", sólo para IAM. Para EIC, ECO, y ECAV las tasas de mortalidad en estos dos grupos de edad disminuyen solo hasta el 2000, con valores relativamente estables o incluso repuntes hacia el incremento entre el 2000 y el 2005 (tabla 5 y figuras 3 y 4).

Tabla 5. Tasas de mortalidad específicas por grupos de edad

\begin{tabular}{|c|c|c|c|c|c|c|c|c|}
\hline & \multicolumn{4}{|c|}{ Hombres } & \multicolumn{4}{|c|}{ Mujeres } \\
\hline Inf. agud, miocardio (IAM) & 1990 & 1995 & 2000 & 2005 & 1990 & 1995 & 2000 & 2005 \\
\hline $0-29$ & 1,2 & 1,5 & 1,3 & 0,7 & 0,3 & 0,3 & 0,0 & 0,5 \\
\hline 30 a 49 & 40,6 & 34,5 & 27,0 & 21,0 & 18,5 & 12,1 & 7,8 & 6,8 \\
\hline 50 a 59 & 185,8 & 161,8 & 140,3 & 103,7 & 105,9 & 72,6 & 47,9 & 46,3 \\
\hline 60 a 69 & 490,5 & 363,7 & 264,5 & 228,1 & 280,2 & 233,8 & 141,7 & 110,7 \\
\hline 70 a 79 & 1021,6 & 864,0 & 504,0 & 448,2 & 736,8 & 571,0 & 339,6 & 308,6 \\
\hline $80+$ & 2932,3 & 2288,5 & 1089,6 & 806,2 & 2601,8 & 1912,3 & 916,0 & 692,2 \\
\hline \multicolumn{9}{|l|}{ Enf. isq. corazón (EIC) } \\
\hline $0-29$ & 1,2 & 1,6 & 1,5 & 0,7 & 0,3 & 0,3 & $0-29$ & 1,2 \\
\hline 30 a 49 & 42,9 & 36,2 & 30,0 & 24,6 & 20,2 & 13,4 & 9,1 & 8,5 \\
\hline 50 a 59 & 212,5 & 183,7 & 173,8 & 138,3 & 121,3 & 88,1 & 67,3 & 68,1 \\
\hline 60 a 69 & 586,5 & 456,5 & 375,0 & 361,7 & 359,3 & 319,0 & 236,3 & 204,1 \\
\hline 70 a 79 & 1376,1 & 1223,9 & 924,9 & 921,5 & 1057,8 & 870,6 & 663,2 & 681,6 \\
\hline $80+$ & 4728,0 & 3896,4 & 2575,1 & 2487,4 & 4313,6 & 3475,2 & 2357,8 & 2357,5 \\
\hline \multicolumn{9}{|l|}{ Enf. del corazón (ECO) } \\
\hline $0-29$ & 3,8 & 5,3 & 4,4 & 2,8 & 2,8 & 3,6 & 1,9 & 2,3 \\
\hline 30 a 49 & 54,5 & 49,9 & 44,0 & 39,1 & 33,0 & 23,6 & 19,9 & 17,0 \\
\hline 50 a 59 & 249,2 & 220,9 & 228,3 & 191,6 & 157,3 & 122,4 & 100,9 & 108,0 \\
\hline 60 a 69 & 668,9 & 551,0 & 478,3 & 479,1 & 435,1 & 391,0 & 325,6 & 292,5 \\
\hline 70 a 79 & 1556,1 & 1446,8 & 1166,5 & 1218,5 & 1226,6 & 1054,6 & 883,9 & 943,9 \\
\hline $80+$ & 5398,7 & 4765,5 & 3395,6 & 3462,3 & 4954,8 & 4264,1 & 3060,5 & 3186,1 \\
\hline \multicolumn{9}{|l|}{ Enf, cardiovasculares (ECAV) } \\
\hline $0-29$ & 5,3 & 6,3 & 5,1 & 4,0 & 4,1 & 4,4 & 2,9 & 3,0 \\
\hline 30 a 49 & 76,8 & 66,7 & 59,3 & 52,4 & 52,4 & 39,8 & 34,0 & 26,5 \\
\hline 50 a 59 & 340,9 & 297,6 & 306,6 & 261,3 & 231,2 & 186,0 & 159,6 & 160,3 \\
\hline 60 a 69 & 903,5 & 784,5 & 688,7 & 685,0 & 637,7 & 589,6 & 490,5 & 450,3 \\
\hline 70 a 79 & 2247,8 & 2148,8 & 1846,8 & 1901,5 & 1828,8 & 1667,5 & 1425,6 & 1488,5 \\
\hline $80+$ & 7977,0 & 7342,6 & 5674,5 & 5448,9 & 7600,8 & 6810,2 & 5330,0 & 5234,5 \\
\hline
\end{tabular}

Ordúñez ${ }_{1}^{1}$ dice además, que la mortalidad por ECEV muestra tres etapas bien definidas: una de rápido descenso de las tasas entre 1970 y 1979, una de muy ligero descenso en las tasas entre 1980 y 1999, y una última de rápido descenso entre 2000 y 2003 . En realidad, nos parece que esta última etapa es demasiado corta (solo 3 años) como para declararla una etapa per se; más bien observamos en la figura 5 del articulo de Orduñez, ${ }^{1}$ que después del rápido descenso entre 1970 y 1979, desde 1980 las tasas de mortalidad por ECEV han tenido pequeñas oscilaciones pero con una tendencia general a disminuir ligeramente. Esto se confirma con nuestros resultados cada 5 años (tabla 1 ) que muestran tasas de mortalidad de 65,$4 ; 62,0 ; 56,4$ y 55,6 en 1990, 1995, 2000 y 2005, respectivamente. 
El análisis de los AVPP por muertes prematuras es especialmente relevante debido a que este indicador muestra de manera directa el impacto negativo que tiene la mortalidad por las distintas causas sobre la esperanza de vida en Cuba, tema muy poco abordado en las publicaciones científicas del país. ${ }^{10}$

En un estudio reciente (Seuc AH. Evolution of Cuban Disease Burden at 5-year intervals: 1990-2005. Pendiente de publicación) encontramos que, como promedio, entre 1990 y 2005, las ECO son responsables aproximadamente de $22 \%$ de todos los AVPP en el país, mientras que la correspondiente cifra para Cáncer es muy similar, $21 \%$; si consideramos ECAV entonces la cifra es $33 \%$, es decir que el aporte conjunto de ECAV y Cáncer en términos de AVPP es de más de $50 \%$ como promedio para el periodo 1990-2005. A esto debemos añadir y reiterar ${ }^{11}$ (Seuc AH. Evolution of Cuban Disease Burden at 5-year intervals: 1990-2005. Pendiente de publicación), que las tendencias durante el periodo para estas dos grandes causas son opuestas; mientras que las tasas de AVPP por ECO han disminuido, las de Cáncer han aumentado, lo que provoca, reiteramos, que desde 1999 Cáncer sea la principal causa de AVPP en Cuba. Otro dato relevante que se presenta en el citado trabajo de Seuc $\mathrm{AH}$, no publicado aún, es que como promedio, durante 1990-2005, las muertes por ECO ocurrieron en personas que son entre 3 y 4 años más viejas que las correspondientes muertes por Cáncer.

Si bien estas cifras del impacto de la mortalidad por ECO (ECAV) y Cáncer son altisonantes, debemos tener presente que esto no quiere decir necesariamente que la principales reservas para el aumento de la esperanza de vida en Cuba radiquen en estas dos grandes causas. Para profundizar en ese tema habría que distinguir, de toda esa mortalidad prematura, qué parte es evitable y qué parte no, en base al contexto de recursos tecnológicos, humanos y organizativos con que cuenta, en la actualidad y en el futuro previsible, el Sistema Nacional de Salud y la sociedad en su conjunto.

Si entramos al detalle del comportamiento de las tasas de AVPP para las causas específicas estudiadas dentro de ECAV, podemos decir que el hecho de que no hayamos encontrado (casi) diferencias en los patrones de las tasas de mortalidad y las tasas de AVPP, globalmente y desglosadas por sexo (tablas 1, 2 y 3), es reflejo de que los cambios observados, incrementos o decrementos, en las tasas de mortalidad se han producido de manera aproximadamente proporcional en los distintos grupos de edades, tanto en hombres como mujeres.

El impacto negativo sobre la esperanza de vida de ECAV y sobre todo del IAM se ha reducido de manera apreciable en el periodo estudiado. Es probable que la mayor afectación a la esperanza de vida en el país al momento de publicarse este trabajo, dentro de todas las ECAV, se deba ya a OEI, la cual ha incrementado sus tasas de AVPP de manera ligera pero sostenida entre 1995 y 2005 (tabla 1 y figura 2), sin embargo, la utilidad de este resultado se ve limitada por el carácter inespecífico de OEI.

Algunas causas que mostraron tendencias positivas (reducciones en sus tasas de mortalidad y de AVPP) en el periodo estudiado, mostraron al mismo tiempo desaceleraciones en esas reducciones entre el 2000 y el 2005; ejemplo notable el IAM. Al mismo tiempo, algunas otras causas en los grupos de edades mayores mostraron incluso repuntes al incremento en sus tasas entre el 2000 y el 2005, revirtiendo así decrementos sostenidos entre 1990 y el 2000; ejemplo notable de esta situación es ECO. Dado que los resultados en 1990 podemos considerarlos como reflejo de la situación cubana justo antes del Período Especia", las comparaciones con lo ocurrido en 1995, 2000 y 2005 podrían ser, esencialmente,

http://scielo.sld.cu 
estimaciones de los efectos a distintos plazos de los cambios en los estilos de vida que impuso este período, ${ }^{25}$ de donde:

- Se confirmaría que la "paradoja" del Periodo Especial es real; hubo efectos positivos en la mortalidad por algunas ECAV, disminución marcada en las tasas ajustadas de mortalidad en la segunda década de los $90,{ }^{1}$ como resultado bastante probable de este período, a pesar de todas las calamidades que representó para los planes de vida de los cubanos; esta conclusión se apoya fundamentalmente en las reducciones simultáneas que se produjeron inmediatamente después de 1990 en la incidencia y prevalencia de factores de riesgo como ingestión diaria per capita de calorías, obesidad, y sedentarismo, que se documentan. ${ }^{26}$

- La influencia positiva del Período Especial sobre la mortalidad por algunas causas dentro de las ECAV, al parecer, se ha diluido significativamente entre el 2000 y el 2005. Es posible que después del 2005 se estén dando ya incrementos en las tasas de mortalidad de algunas causas que hasta ese año estaban disminuyendo o permanecían relativamente estables, lo que representaría el fin de la "paradoja" aproximadamente 15 años después de su inicio en 1990.

Finalmente se puede concluir que dentro de las ECO, las EIC excluyendo IAM (es decir OEI) son la segunda causa de muerte, después de IAM, durante el periodo estudiado 1990-2005; al momento de publicarse este trabajo (2009-2010) probablemente OEI haya sobrepasado ya a IAM como primera causa de muerte y de AVPP. Sin embargo, no puede descartarse que este nuevo escenario se deba, en parte, a que algunas muertes por IAM se estuvieron clasificando sistemática y erradamente dentro de OEI.

Durante el periodo 1990-2005 el peso de la mortalidad por EIC dentro de la mortalidad total en el país ha sido importante pero ha ido disminuyendo sostenidamente; en 1990 representaba el $25 \%$ de la mortalidad total, mientras que en el 2005 no llegaba al $20 \%$.

Las tasas de mortalidad y de AVPP por EI durante el periodo 1990-2005 fueron las más bajas de entre todas las causas consideradas en este estudio, pero se debieran explorar las razones por las cuales se observaron incrementos muy notables entre el 2000 y el 2005.

El procedimiento estándar a nivel internacional para el cálculo de los AVPP toma como referencia la esperanza de vida por grupos quinquenales para alguna población de referencia. Hacerlo tomando como referencia una cifra fija (la esperanza de vida al nacer) provoca importantes subestimaciones y distorsiones en los valores de las tasas resultantes. Por ejemplo, la tasa $(\times 1000)$ de AVPP por ECAV calculada de la primera forma (la correcta) fue algo más de dos veces la cifra calculada de la segunda forma, aproximadamente 25 y 11 respectivamente, en el año 2003 para Cuba.

Se pone de manifiesto la necesidad de explorar las razones por las que en Cuba la brecha entre hombres y mujeres con respecto a la mortalidad por ECAV y en particular por EIC, es más pequeña que la que usualmente se observa en países desarrollados. Los resultados de este trabajo sugieren que existe un potencial de incremento de salud en las mujeres cubanas que no se ha estado aprovechando suficientemente.

Todo parece indicar que los cambios en los estilos de vida a los que nos obligó el Periodo Especial después de 1990 tuvieron repercusión positiva en las tasas de 
mortalidad y de AVPP por algunas causas dentro de las ECAV en Cuba; hay indicios sin embargo, de que a partir del 2000 aproximadamente tales efectos positivos, desafortunadamente, empezaron a diluirse sustancialmente.

El aumento de la carga por Cáncer que acompaña a la disminución de la carga por ECO, y el aumento de la carga por OEI que acompaña a la disminución de la carga por IAM, muestra la importancia de que los análisis de la mortalidad por alguna causa (o grupo de causas) específica pongan en contexto la causa de interés con respecto a algunas de las restantes causas "competitivas" relevantes. ${ }^{27,28}$ En el caso que nos ocupa, que la mortalidad por ECO está disminuyendo desde hace por lo menos 15 años $^{11}$ y que en relativamente poco tiempo será sobrepasada por la mortalidad por Cáncer, es una interacción importante que debiera mencionarse pues propicia un análisis acerca de si la mencionada disminución en ECO se debe a un mejor trabajo (de promoción, prevención y atención) relacionado con esta enfermedad, o a un peor trabajo (de promoción, prevención y atención) relacionado con el Cáncer, o a una combinación de estos dos factores. Lástima que la principal causa que compita con ECO sea Cáncer, una causa de muerte demasiado heterogénea con respecto a su etiología y factores de riesgo.

\section{REFERENCIAS BIBLIOGRÁFICAS}

1. Orduñez P. Enfermedades cardiovasculares en Cuba: determinantes para una epidemia y desafíos para la prevención y control. Rev Cubana Salud Pública[serie en Internet]. 2005 Dic [citado 2010 Sep 30];31(4). Disponible en:

http://scielo.sld.cu/scielo.php?script=sci arttext\&pid=S086434662005000400002\&lng=es

2. Gallardo U. Impacto de la Mortalidad por Enfermedades Vasculares Periféricas, Cuba 2000. Rev Cubana Angiol Cirugía Vas [serie en Internet]. 2005 [citado 2010 Sep 30];6(1). Disponible en: http://bvs.sld.cu/revistas/ang/vol6 1 05/ang01105.htm

3. Debs G. Prevalencia de factores de riesgo coronario en "10 de Octubre"; su evolución a los 5 años. Rev Cubana Cirugía Cardiovasc. 2001;15(1):14-20.

4. Ramos BN, Mendez G. Comportamiento de factores de riesgo en las enfermedades del corazón y cerebrovascular. Rev Cubana Hig Epidemiol. $2006 ; 44(3)$.

5. Oliva JE. Enfermedad cerebrovascular: comportamiento en el Hospital Docente "Dr. Salvador Allende" durante 1997. Rev Cubana Invest Biomed. 2001;20(3):197201.

6. Batista R. Sistema de vigilancia de enfermedades no transmisibles en Cuba. Rev Cubana Hig Epidemiol. 2000;38(2):77-92.

7. Dominguez E, Seuc AH. Esperanza de vida ajustada por algunas enfermedades crónicas no transmisibles. Rev Cubana Hig Epidemiol [serie en Internet]. 2005 Ago [citado 2010 Sep 30];43(2). Disponible en:

http://bvs.sld.cu/revistas/hie/vol43 2 05/hie06205.htm

8. Madrazo JM, Madrazo AM. Actuales factores de riesgo aterogénico en la génesis de la cardiopatía isquémica: problemática epidemiológica mundial. Rev Cubana

http://scielo.sld.cu 
Invest Biomed [serie en Internet]. 2005 Jun [citado 2010 Sep 30];24(2). Disponible en: http://scielo.sld.cu/scielo.php?script=sci arttext\&pid=S0864$\underline{03002005000200010 \& \operatorname{lng}=\mathrm{es}}$

9. Rodríguez Ojea A. La transición nutricional y las enfermedades cardiovasculares en la década de los noventa. Acta médica. 2003;11(1):26-37.

10. Coutin G, Borges J, Batista R, Feal P. El control de la hipertensión arterial puede incrementar la esperanza de vida: verificación de una hipótesis. Rev Cubana Med. $2001 ; 40(2): 103-8$.

11. Seuc AH, Domínguez E, Gallardo U, García RM, López L, González E. Mortalidad y años de vida potencial perdidos por muertes prematuras en mujeres cubanas: 1990, 1995 y 2000. Rev Cubana Salud Pública [serie en Internet]. 2004 Dic [citado 2010 Sep 30]; 30(4). Disponible en: http://scielo.sld.cu/scielo.php?script=sci arttext\&pid=S086434662004000400002\&lng=es

12. Torres RM, Gran MA. Panorama de la salud del adulto mayor en Cuba. Rev Cubana Salud Pública [serie en Internet]. 2003 Sep [citado 2010 Sep; 30];29(3). Disponible en: http://scielo.sld.cu/scielo.php?script=sci arttext\&pid=S086434662003000300002\&lng=es

13. Rodríguez LE, Martínez L, Pría MC, Menéndez J. Prevalencia referida de enfermedades no transmisibles en adultos mayores: Ciudad de La Habana, 2000. Rev Cubana Hig Epidemiol [serie en Internet]. 2004 Abr [citado 2010 Sep 30]; 42(1). Disponible en:

http://scielo.sld.cu/scielo.php?script=sci arttext\&pid=S156130032004000100006\&lng=es.

14. Ramírez M, Debs G. El problema de la cardiopatía isquémica en Cuba. RESUMED. 2001;14(4):160-1.

15. Dueñas A. Programa Nacional de Prevención, Diagnóstico, Tratamiento y Rehabilitación de la Cardiopatía Isquémica. RESUMED. 2001;14(4):157-9.

16. Murray CJL, Ezzati M, Lopez AD, Rodgers A, Hoorn SV. Comparative quantification of health risks: Conceptual framework and methodological issues. Populat Health Metric. 2003;1:1.

17. Oficina Nacional de Estadística (ONE). Anuario Demográfico de Cuba Centro de Estudios de Población y Desarrollo. La Habana: ONE; 2000.

18. Dirección Nacional de Estadística (DNE). Anuario Estadístico de Salud 2000. La Habana: DNE, MINSAP; 2001.

19. Dirección Nacional de Estadística (DNE). Anuario Estadístico de Salud 2005. La Habana: DNE, MINSAP; 2006.

20. Dirección Nacional de Estadística (DNE). Anuario Estadístico de Salud 2003. La Habana: DNE, MINSAP; 2004.

21. Dirección Nacional de Estadística (DNE). Anuario Estadístico de Salud 2007. La Habana: DNE, MINSAP; 2008.

http://scielo.sld.cu 
22. Pilote L. A comprehensive view of sex-specific issues related to cardiovascular disease. CMAJ. 2007;176(6):S1-44.

23. Lehto HR. Are coronary event rates declining slower in women than in men evidence from two population-based myocardial infarction registers in Finland? BMC Cardiovasc Disorders. 2007; 7:35.

24. WHO. World Health Statistics 2006. Geneva, WHO [sitio en Internet]. [citado 19 Dic 2006]. Disponible en: http://www.who.int/whosis/whostat2006.pdf

25. Unal B. Life-Years Gained From Modern Cardiological Treatments and Population Risk Factor Changes in England and Wales, 1981-2000. Am J Public Health. 2005;95:103-8.

26. Franco M. Impact of Energy Intake, Physical Activity, and Population-wide Weight Loss on Cardiovascular Disease and Diabetes Mortality in Cuba, 1980-2005. Amer J Epidemiol. 2007;166(12):1374-80.

27. Bursi F. Heart Disease and Dementia: A Population-based Study. Amer J Epidemiol. 2005;163(2).

28. Kim HT. Cumulative Incidence in Competing Risks Data and Competing Risks Regression Analysis. Clin Cancer Res. 2007;13(2).

Recibido: 10 de febrero de 2010.

Aprobado: 30 de julio de 2010.

Armando H. Seuc. Instituto de Higiene, Epidemiología y Microbiología (INHEM). Infanta No. 1158 e/ Clavel y Llinás. Centro Habana 10300. La Habana, Cuba. Correo electrónico: seuca@who.int; metodoli@infomed.sld.cu 